\title{
A PLATE TEST FOR NITRATE REDUCTION
}

\author{
BY \\ G. T. COOK \\ From the Public Health Laboratory, Oxford
}

(RECEIVED FOR PUBLICATION JUNE 9, 1950)

Some effects of nitrates in bacteriological media are described in the accompanying paper (Cook and Knox, page 356). Among the observations made was that of a dark zone round colonies of nitrate-reducing organisms on blood agar containing potassium nitrate. Further experiments along these lines were carried out and resulted in the present plate test for nitrate reduction.

\section{Method}

The test is based on the oxidation of haemoglobin to methaemoglobin in the presence of nitrites and is carried out by making a stab inoculation into a blood agar medium containing potassium nitrate.

Potassium nitrate may be either (1) incorporated in the blood agar medium, or (2) added to it before use by means of a " nitrate strip."

1. A $20 \%$ solution of potassium nitrate (sterilized by steaming for one hour) is added to $6 \%$ horse blood tryptic digest heart agar to give a final concentration of $0.1 \%$ potassium nitrate. The plates are poured unlayered.

2. A blotting-paper strip containing potassium nitrate is placed on the centre of a layered $6 \%$ horse blood digest heart agar plate at the time of inoculation. The strips are prepared by soaking sheets of Ford's blotterettes ("Ford 428 Mill ") in a warm $40 \%$ solution of potassium nitrate. The sheets are hung up to dry in a $37^{\circ} \mathrm{C}$. incubator and then cut up into small strips of approximately $16 \times 10 \mathrm{~mm}$. The strips are put into testtubes and autoclaved for 10 minutes at $10 \mathrm{lb}$.

Two cultures can be conveniently tested on the same plate, each being stabbed into the centre of opposite halves of the medium. The results are read after 18-24 hours' incubation at $37^{\circ} \mathrm{C}$., but plates which are negative should be reincubated for a further 24 hours. A positive result is indicated by a dark green-brown zone surrounding the site of inoculation and most easily seen against a white background or by transmitted light (Figs. 1, 2, and 3). The dark zone is due to the oxidation of haemoglobin within the red cell to methaemoglobin, the presence of which was confirmed by spectroscopic examination. This change is due to the gradual diffusion of nitrite produced by the organisms from potassium nitrate. The diameter of the zone varies with the time of incubation, depth of medium, and nature of the organism, but is usually between 2 and $4 \mathrm{~cm}$. It is not advisable to inoculate more than two cultures on plates of approximately $9 \mathrm{~cm}$. diameter as, with three or more positive strains, the extensive diffusion of nitrite may result in discoloration throughout the medium.

The plates are normally incubated aerobically, though better results with Haemophilus and Pseudomonas strains have been obtained on anaerobic incubation. Immediate inspection of the plate on removal from the jar shows the inoculated area surrounded by a zone of cherry red colour. On exposure to air this zone rapidly darkens, and after 15-20 minutes on the bench the colour has changed to the characteristic dark greenishbrown of methaemoglobin. 
FIG. 1.

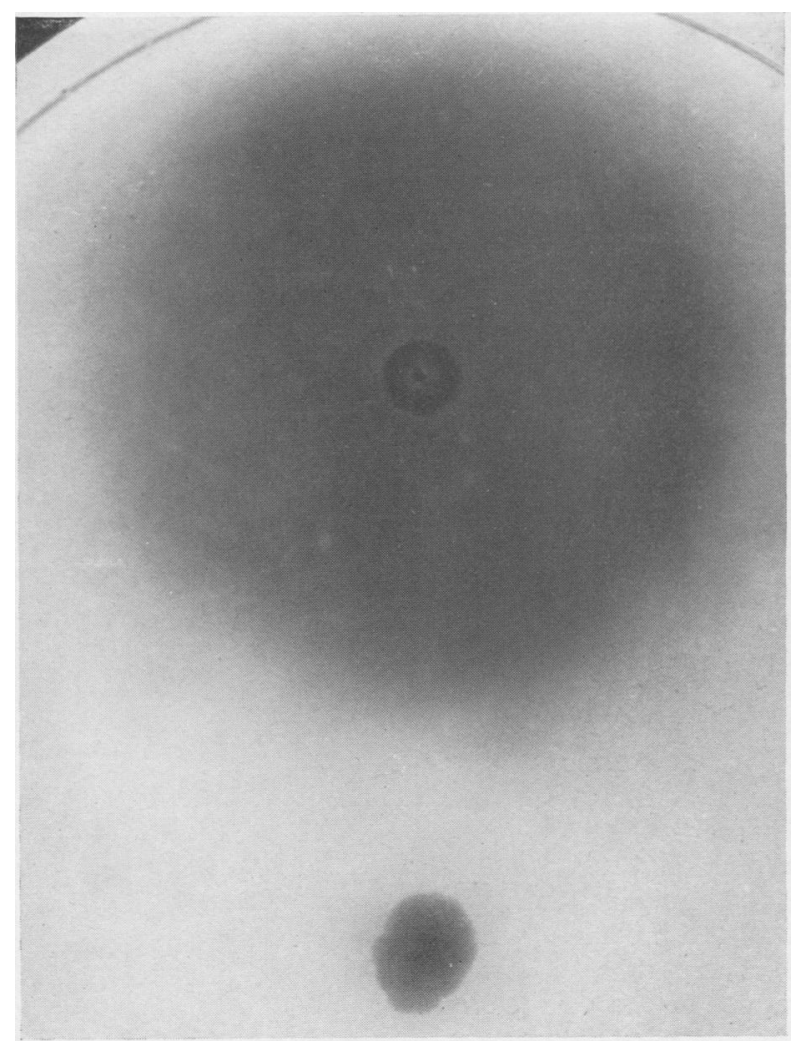

Fig. 2.

FIG. 1.-Stab inoculation of Bact. coli on $0.1 \%$ nitrate plate incubated for 24 hours.

FIG. 2.-Stab inoculation of $C$. diphtheriae var. gravis (above) and $C$. diphtheriae var. intermedius (below) on $0.1 \%$ nitrate plate incubated for 24 hours.

FIG. 3.-Stab inoculation of Staph. aureus (above) and a starch-fermenting gelatin-liquefying corynebacterium (below) on $0.1 \%$ nitrate plate incubated for 24 hours. The corynebacterium does not reduce nitrate.

Fig. 3. 


\section{Comparison of the Plate Test with Nitrate Broth}

One hundred and seven strains from 13 genera have bsen tested for nitrate reduction by each of the two methods described in this paper and after growth in $0.1 \%$ nitrate broth. The plates were examined after 24 and 48 hours' aerobic incubation, and the broth cultures were tested for the presence of nitrite by the GriessIlosvay method (Wilson and Miles, 1946) after five days' incubation. Negative broth cultures were tested for the presence of unreduced nitrate by the addition of zinc powder (ZoBell, 1932).

Similar results were obtained with each of the three methods: 71 strains were nitrate reducers and 36 did not reduce nitrate. All but one of the 71 strains able to reduce nitrate gave a positive result by each of the plate methods after 24 hours. The exception, a diphtheroid, did so after 48 hours. Weak results by the plate methods were shown by some Pseudomonas and Haemophilus strains, though these strains always gave a satisfactory positive result on anaerobic incubation. In fact, each of the six strains of $H$. influenzae and $H$. para-influenzae included in the series gave a more definite result by the plate test carried out anaerobically than by the Griess-Ilosvay method.

\section{Discussion}

Certain points concerning the optimal concentration of nitrate in the medium and the method of inoculation are of special interest. It was found that, using potassium nitrate in a concentration of only $0.05 \%$ in the medium or of $10 \%$ in the strips, negative results were obtained with certain vigorous nitrate-reducing coliform and staphylococcal strains. Some of these strains also gave a negative GriessIlosvay test after 24 hours though positive at six hours, and the negative results on the plates may be due to rapid reduction of nitrite to substances which failed to discolour the medium. This difficulty was overcome by increasing the nitrate in the medium and the strips to $0.1 \%$ and $40 \%$ respectively and so ensuring a concentration of nitrite sufficient to produce a visible colour change in the cell haemoglobin.

It is also important that the method of inoculation of the plate should be a stab into the medium rather than a surface streak. Although most of the positive strains also reduced nitrate when inoculated on to the surface of the medium, some strains of Haemophilus and two of Corynebacterium diphtheriae var. intermedius failed to do so. Some degree of anaerobiosis appeared to be necessary for nitrate reduction by these organisms, all of which gave satisfactory results aerobically with a stab inoculation or with a surface inoculation provided the plate was incubated anaerobically. A positive result obtained by placing a " nitrate strip" across a streak inoculation of $C$. diphtheriae var. intermedius on a blood agar plate was shown to be due to the semi-anaerobic conditions produced under the strip.

It is well recognized that false negative results may be obtained when a nitrate broth culture is tested for the presence of nitrite by the Griess-Ilosvay method, as some species are able to reduce nitrites almost as rapidly as they are formed. A number of methods have been suggested in order to overcome this difficulty, and it is possible, for instance, to test for nitrite reduction products such as hydroxylamine, ammonia, or free nitrogen in the medium, though certain precautions have to be taken before reliable results are obtained (Conn, 1936). A more satisfactory and simple method is the zinc dust test for residual nitrate described by ZoBell 
(1932), who recommends that it should invariably be made in conjunction with the test for nitrites when the latter is negative. One of the advantages of the plate test for nitrate reduction is that the cell haemoglobin acts as a sensitive and irreversible indicator of the presence of nitrites and so identifies as nitrate reducers organisms capable of removing nitrites in a fluid medium by further reduction. Of the 71 strains which gave a positive nitrate test on the plate, only 45 were positive by the Griess-Ilosvay technique. The remaining 26 cultures, however, were all negative to the zinc dust test.

The plate test is easy to carry out and the results can be read by simple inspection of the plate, in many cases within a few hours of inoculation. "Nitrate strips" kept on the bench maintain their activity for several weeks and can be employed with the ordinary horse blood digest agar plate. This is a point of some practical importance for the routine bacteriologist, who will probably seldom need to test for nitrate reduction. It is possible that a simpler method of performing the test may increase the usefulness of this procedure in diagnostic bacteriology. In this laboratory the rapidity of the plate test has proved of assistance in distinguishing starchfermenting gelatin-liquefying corynebacteria from $C$. diphtheriae var. gravis (Jebb, 1948).

\section{Summary}

A plate test for nitrate reduction is described. The test is based on the oxidation of haemoglobin to methaemoglobin by nitrites and is carried out on a blood agar medium containing potassium nitrate. Colonies of nitrate-reducing organisms are surrounded by an area of green discoloration.

The plate test is simple to carry out and has some advantages over the GriessIlosvay method of testing for nitrate reduction.

My thanks are due to Mr. H. A. Tarr for the photographs.

\section{REFERENCES}

Conn, H. J. (1936). J. Bact., 31, 225.

Jebb, W. H. H. (1948). J. Path. Bact., 60, 403.

Wilson, G. S., and Miles, A. A. (1946).' Topley and Wilson's Principles of Bacteriology and Immunity, 3rd ed., vol. 1, p. 368 . London.

ZoBell, C. E. (1932). J. Bact., 24, 273. 